Portland State University

PDXScholar

$5-1-2008$

\title{
Unipolar Transport and Interface Charge Transfer in Nanostructured CdTe/polymer Hybrid Films
}

\author{
Rolf Könenkamp \\ Portland State University, rkoe@pdx.edu \\ Robert Meier \\ Portland State University \\ Robert Campbell Word \\ Portland State University \\ Athavan Nadarajah \\ Portland State University
}

Follow this and additional works at: https://pdxscholar.library.pdx.edu/phy_fac

Part of the Physics Commons

Let us know how access to this document benefits you.

Citation Details

Meier, Robert, Word, Robert C., Nadarajah, Athavan and Könenkamp, Rolf. (2008). Unipolar transport and interface charge transfer in nanostructured CdTe/polymer hybrid films. Physical Review B, 77, 195314

This Article is brought to you for free and open access. It has been accepted for inclusion in Physics Faculty Publications and Presentations by an authorized administrator of PDXScholar. Please contact us if we can make this document more accessible: pdxscholar@pdx.edu. 


\title{
Unipolar transport and interface charge transfer in nanostructured CdTe/polymer hybrid films
}

\author{
Robert Meier, Robert C. Word, Athavan Nadarajah, and R. Könenkamp* \\ Physics Department, Portland State University, Portland, Oregon 97201, USA \\ (Received 30 January 2008; revised manuscript received 15 April 2008; published 14 May 2008)
}

\begin{abstract}
We report detailed photoconductance measurements on hybrid semiconductor films consisting of CdTe nanoparticles in a poly[2-methoxy-5-(2'-ethyl-hexyloxy)-1,4-phenylene vinylene (MEH-PPV) matrix. Our results show that a staggered band alignment between organic and inorganic semiconductors produces an efficient separation of photoexcited electron-hole pairs and promotes a unipolar transport in each of the semiconductors. Hole drift mobilities in the MEH-PPV film and electron drift mobilities in the CdTe nanoparticle network are determined. We find clear evidence for the transport in the two phases of the hybrid film to be electrically uncoupled, and we conclude that these properties can lead to improved electron transport properties.
\end{abstract}

DOI: 10.1103/PhysRevB.77.195314

PACS number(s): 73.40.-c, 72.80.Le, 72.80.Tm, 73.50.Pz

Organic/inorganic hybrid films are presently being studied for the fabrication of novel device structures in solar cells, detectors, sensors, and light-emitting diodes. ${ }^{1-5}$ In some of these applications, the inorganic nanoparticles serve as sensitizing agents for organic films. This type of hybrid structure creates a broad spectrum of new technical possibilities, which arise from the interesting optical properties of nanoparticles and the unique mechanical characteristics of organic semiconductors. Simple band gap tailoring, large interface areas, flexibility, and simple preparation conditions are some of the main advantages of these hybrid films. These properties have raised expectations for new device structures in the areas of energy conversion, lighting, and sensing. At the present stage, however, there are still a number of challenges to be addressed that are related to the charge transport in these materials. The electron transport in the bulk, as well as the charge transfer across the organic/inorganic interface, needs further study before significant progress in applications can be expected.

We have carried out a detailed photoconductance study on a hybrid material system consisting of nanosize $\mathrm{CdTe}$ particles randomly distributed in a thin poly[2-methoxy-5-(2'-ethyl-hexyloxy)-1,4-phenylene vinylene (MEH-PPV) film. We used a highly sensitive interference grating technique to characterize the electron transport in these films. Our results show that the electron-hole pairs generated in CdTe are effectively separated across the phase boundary in the hybrid films. The holes are injected from CdTe into MEH-PPV, while the photoexcited electrons remain on the CdTe nanoparticles. After injection, the carriers are electrically decoupled from each other and both carrier types become majority carriers in the two components of the hybrid film. When the nanoparticles are not connected and a transport path is not established, the electrons in CdTe do not contribute to the transport, of course. In this case, the photoconductance observed in the films is due to the excess holes in the MEH-PPV film, which have been photogenerated in the CdTe nanoparticles and transferred across the organic and/or inorganic interface. We were able to experimentally determine the resulting hole mobilities. When the CdTe particle density is sufficiently high and an interconnected CdTe particle network is established, the photoexcited electrons can be transported within the nanoparticle network. We were also able to determine the mobilities for this mixed transport case with the electron transport in CdTe and the hole transport in MEH-PPV. Surprisingly, in this case, the two carrier ensembles exhibit unipolar rather than ambipolar carrier transport characteristics, i.e., the two phases of the hybrid film are not electrically coupled. These transport conditions are supportive of long recombination times and large transport distances for excess carriers. In most cases, these conditions are preferable over the conditions encountered in homogeneous materials, wherein excess electrons and holes form an ambipolar space charge-neutral carrier gas in which transport velocity and transport distances are limited by the coupling. Our results also suggest that the CdTe particles, despite being $p$-type, show electron-dominated transport when incorporated in the hybrid films.

Uncapped, ligand-free CdTe particles were obtained by nanomilling of high-purity undoped CdTe crystals obtained from $5 \mathrm{~N}$ Inc. The milling was performed for $5 \mathrm{~h}$ at Hampton University, VA. ${ }^{6}$ Scanning electron micrographs (SEMs) showed that the typical grain diameter achieved in the milling was in the range of 50-250 nm. High-purity MEH-PPV with a molecular weight of $250000 \mathrm{~g} / \mathrm{mol}$ was dissolved in pure chloroform by gentle rocking for $12 \mathrm{~h}$. The CdTe nanoparticle powder was then added to the MEH-PPV/chloroform solution and homogenized by using sonication at a power of $400 \mathrm{~W}$ for $12 \mathrm{~min}$. The resulting suspension was spin coated on alkali-free glass substrates, and the obtained films were provided with Au strip contacts with a separation of $0.33 \mathrm{~mm}$ by using vacuum evaporation through a mask. A similar procedure was used to prepare $\mathrm{CdTe} /$ polystyrene hybrid films. Figure 1 shows the SEMs of two different concentrations of nanoparticles in the MEH-PPV films. For the lower concentration shown in Fig. 1(a), the particles are not interconnected, while for the higher concentration, the micrograph in Fig. 1(b) indicates a sufficient connectivity for transport through the CdTe particle network.

Pure CdTe nanoparticle films were prepared without MEH-PPV by distributing the CdTe nanoparticles on an adhesive insulator surface and applying gentle compaction at pressures of approximately $1 \mathrm{kp} / \mathrm{cm}^{2}$.

The determination of the transport parameters uses a moving interference grating to laterally drive a photocurrent through the hybrid films. We have previously used this mov- 


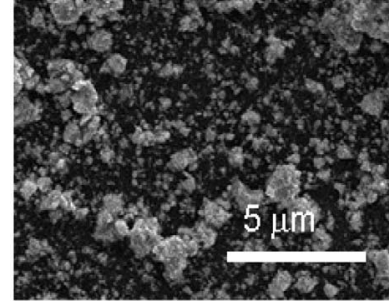

a) b)

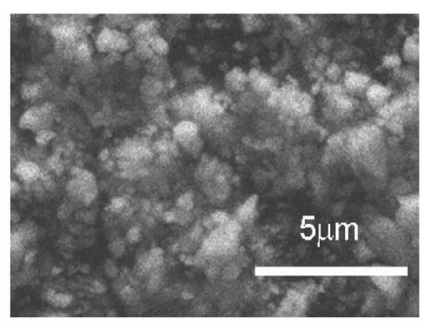

FIG. 1. Scanning electron micrograph of CdTe/MEH-PPV hybrid films: (a) prepared at a weight mixing ratio of 7:10 CdTe/ MEH-PPV; (b) prepared at a weight mixing ratio of 15:10 CdTe/ MEH-PPV. The dark background represents the MEH-PPV film, while the bright area shows the CdTe particles, which are partially agglomerated. The connectivity of the particle network is evident in part (b).

ing photocarrier grating (MPG) technique to characterize low-mobility amorphous and organic semiconductors. ${ }^{7}$ Our present setup uses a $633 \mathrm{~nm}$ beam from a HeNe laser with an area of $\sim 1.33 \mathrm{~mm}^{2}$ and a total power of $0.1 \mathrm{~mW}$. The beam passes through a beam splitter and the two partial beams are then detuned with respect to each other in two acoustooptical modulators. The detuning covers the range $-0.15 \mathrm{MHz}<\Delta<0.15 \mathrm{MHz}$. The partial beams are superimposed at an angle $\alpha$ on the sample surface, producing an intensity interference grating with a repeat distance of $\Lambda$ $=\lambda / 2 \sin (\alpha / 2)$ moving at a velocity of $v=\Lambda \Delta$. Figure 2 shows the experimental setup. The intensity grating gives rise to spatial variations in the electron and hole densities. While the photogenerated hole and electron densities are equal, the kinetic response of the two carrier ensembles to the moving grating is generally different. As a consequence, a partial charge separation along the propagation direction of the grating occurs, which gives rise to a net photocurrent. In homogeneous materials, the photogenerated electron and hole distributions usually remain electrically coupled: When dielectric relaxation occurs on a sufficiently fast time scale, local space charge neutrality provides this coupling. The transport process is then ambipolar and the current direction is determined by the motion of the dominant carrier type.
The current dependence on the grating velocity can be accurately described in terms of the carrier drift, diffusion, and recombination parameters, as described in Refs. 8 and 9. Figure 2(b) shows a typical curve for the MPG-induced photocurrent in a lightly doped semiconductor film under ambipolar transport conditions. At zero and at very high grating velocities, the photocurrent goes to zero. When the grating is not moving, the photogenerated carrier distribution between the two contacts is spatially symmetric and no net photocurrent is observed. At very high grating velocities, the carrier distribution cannot follow the kinetics imposed by the grating and the current is also zero. A single maximum current response occurs in the positive and negative direction when the grating velocity is in the range of the carrier drift and diffusion velocities. From the polarity of the photocurrent signal, the dominant carrier type can be determined, and from a curve fit, accurate values for the drift mobilities of holes and electrons, as well as the carrier recombination lifetime, can be determined. In the present experiments, typical current amplitudes at the maximum were in the range 1-50 fA. The experiments were carried out at wavelengths of 633 and $532 \mathrm{~nm}$. These essentially gave same results. In addition, photoconductivity measurements were carried out to obtain more experimental data for the mobilities and lifetimes from the relation $\Delta \sigma=e\left(\Delta n \mu_{e} \tau+\Delta \mathrm{p} \mu_{p} \tau\right)$, where $\Delta \sigma$ is the photoconductivity, $e$ is the electron charge, $G$ is the generate rate, $\mu_{e}$ and $\mu_{h}$ are the electron and hole drift mobilities in CdTe and MEH-PPV, respectively, and $\tau$ is the recombination lifetime. With these additional measurements, the accuracy for the determination of the transport parameters is in most cases better than $\pm 10 \%$.

Figure 3 shows an estimated energy level diagram for the CdTe/MEH-PPV hybrid system based on recent results from the photoemission measurements for the ionization potentials. We used $5.7 \mathrm{eV}$ for the ionization energy of CdTe from Ref. 10 and $5.66 \mathrm{eV}$ for MEH-PPV from Ref. 11. Since CdTe is undoped, while MEH-PPV is $p$ type, we estimate that a band bending on the order of $0.3 \mathrm{eV}$ will occur at the interface, as shown in Fig. 3. Based on the photoemission data, undoped CdTe and MEH-PPV form a staggered heterojunction in which both the valence band offset and the band bending are supportive of the hole transfer from $\mathrm{CdTe}$ to

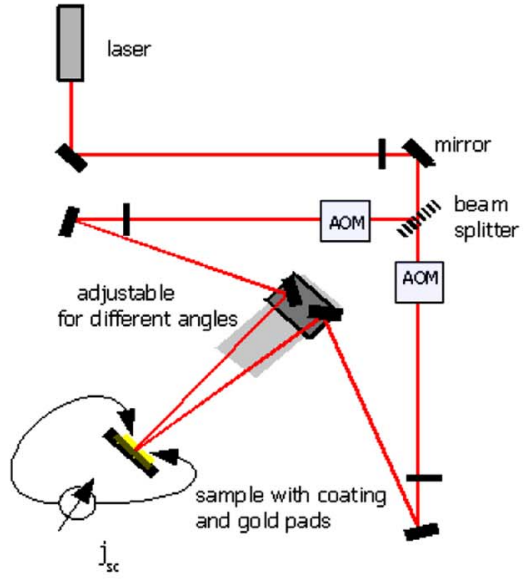

a)

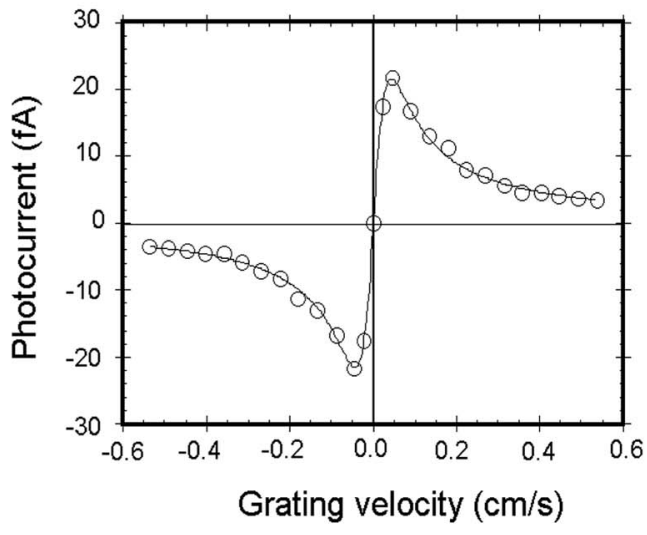

b)
FIG. 2. (Color online) (a) Experimental setup of the moving photocarrier grating technique; (b) Photocurrent response vs grating velocity in the MPG experiment in a lightly doped semiconductor under ambipolar transport conditions. 


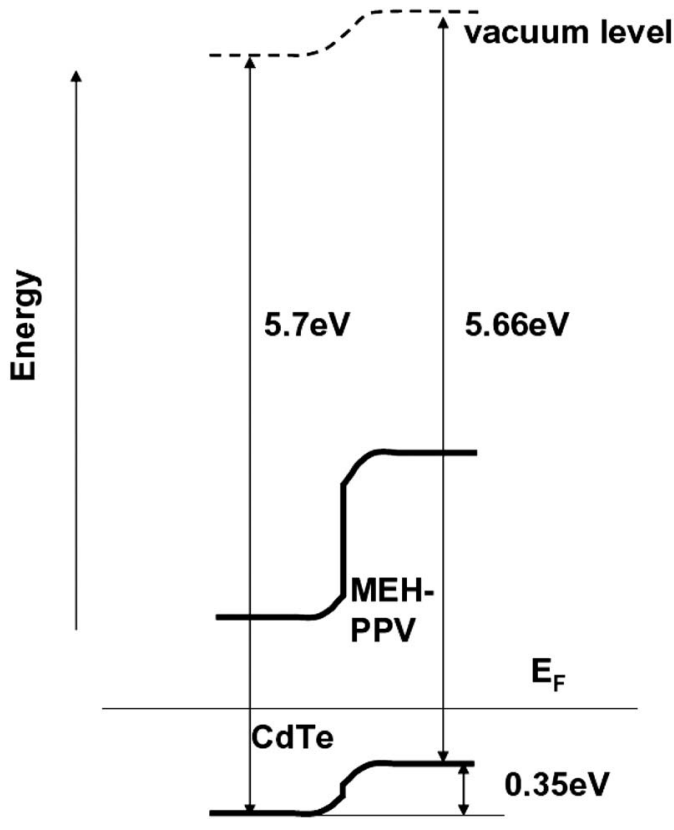

FIG. 3. Schematic band diagram for the staggered band alignment at the CdTe/MEH-PPV interface. The ionization energies are from Refs. 10 and 11.

MEH-PPV. The total energy gain for the hole transfer is of the order of $0.35 \mathrm{eV}$. There is a large energy barrier for the electron transfer from CdTe to MEH-PPV. By using a band gap of $1.5 \mathrm{eV}$ for CdTe and a highest occupied molecular orbital-lowest unoccupied molecular orbital (HOMOLUMO) separation of $2.3 \mathrm{eV}$ for MEH-PPV, this barrier is $1.15 \mathrm{eV}$, which is sufficient to prevent any electron transfer from CdTe to MEH-PPV. The photon energy for the $633 \mathrm{~nm}$ laser light is $1.96 \mathrm{eV}$, allowing photoexcitation in CdTe but not in MEH-PPV due to its larger band gap. The control measurements on pure MEH-PPV films confirmed that there is essentially no absorption and no photoconductance at $\lambda$ $=633 \mathrm{~nm}$.

Since at low particle concentrations the CdTe nanoparticels are not connected and since no carriers are being generated in MEH-PPV, the photocurrents observed in the experiments can be explained only by carrier transfer from CdTe to MEH-PPV. From the photocurrent polarity, it is deduced that the transferred carriers are holes, which is completely consistent with the conclusions drawn from the band diagram in Fig. 3.

Figure 4 shows the MPG results for this case of low nanoparticle densities, wherein the holes transferred to MEH-PPV make up the photocurrent. The heavy solid line in Fig. 4 is obtained from a least-squares fit to a numerical solution of the transport equations with the hole drift mobility and recombination lifetime in MEH-PPV as adjustable parameters. From this procedure, we find $\mu_{h}=5.5 \times 10^{-11} \mathrm{~m}^{2} / \mathrm{V} \mathrm{s}$ and a recombination lifetime $\tau=2.9 \times 10^{5} \mathrm{~s}$ with a relative error of $8 \%$ as represented by the lighter solid lines. The mobility value compares well to the results from the time-of-flight experiments in MEH-PPV, which give $\mu_{h}=10^{-10} \mathrm{~m}^{2} / \mathrm{V} \mathrm{s}$ (Ref. 12) or slightly larger values. ${ }^{13,14}$ As these measurements use electric fields of $\sim 10^{7} \mathrm{~V} / \mathrm{m}$ and the drift mobility

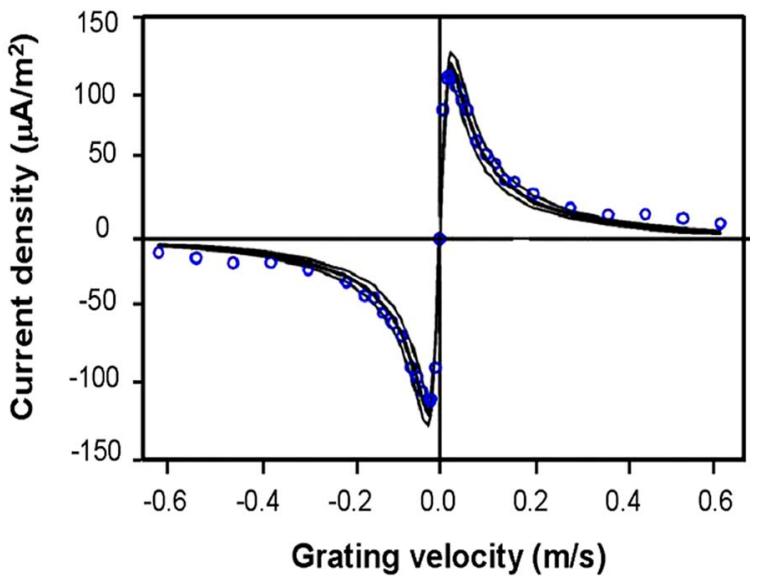

FIG. 4. (Color online) Moving grating results for a CdTe / MEH-PPV film with a low CdTe nanoparticle density, showing hole transport only in the MEH-PPV. The heavy solid line shows a curve fit based on $\mu_{h}=5.5 \times 10^{-11} \mathrm{~m}^{2} / \mathrm{V} \mathrm{s}$ for the hole drift mobility in MEH-PPV and $\tau=2.9 \times 10^{-5} \mathrm{~s}$ for the recombination lifetime. The lighter solid lines are for $+8 \%$ and $-8 \%$ deviations from the drift mobility value.

is field dependent, the time-of-flight results seem consistent with our estimate, which is obtained without externally applied fields. Recombination lifetimes have not been previously reported in MEH-PPV. The obtained lifetime value is consistent with the experimental assumption that the dielectric relaxation occurs faster than the carrier recombination, as the relaxation time is approximately $10^{-7} \mathrm{~s}$ under the experimental conditions.

When higher particle concentrations are used and an interconnected particle network is established, as in Fig. 1(b), the electrons in the CdTe network are no longer localized and are also contributors to the electrical response. For these films, the MPG response signal is quite different from the previous case, as it contains another pair of current peaks occurring at small grating velocities and with polarity opposite to the hole transport peaks, as shown in Fig. 5(a). From the polarity of the new pair of peaks, it is clear that the new current signal is due to the photoexcited electrons. The band diagram shows that this electron response can be attributed only to the electron transport in CdTe. It is therefore concluded that at sufficiently high nanoparticle concentrations, a percolation network is formed and the photogenerated electrons are transported through this network.

The occurrence of the second current peak also shows that the photogenerated electrons and holes are not electrically coupled as one would have expected for a homogeneous thin film. Apparently, the charge transfer across the interface leads to a sufficient spatial separation such that electrical screening and charge neutrality no longer hold between the two carrier ensembles. The experimental data in Fig. 5(a) can then be interpreted as a simple superposition of the hole currents in MEH-PPV and the electron currents in CdTe. A quantitative evaluation gives the electron drift mobility in the CdTe particle network as $\mu_{e}=2.0 \times 10^{-11} \mathrm{~m}^{2} / \mathrm{V} \mathrm{s}$, while the hole drift mobility in MEH-PPV and the recombination lifetime remain as in Fig. 4.

Figure 5(a) thus establishes evidence for two independent 


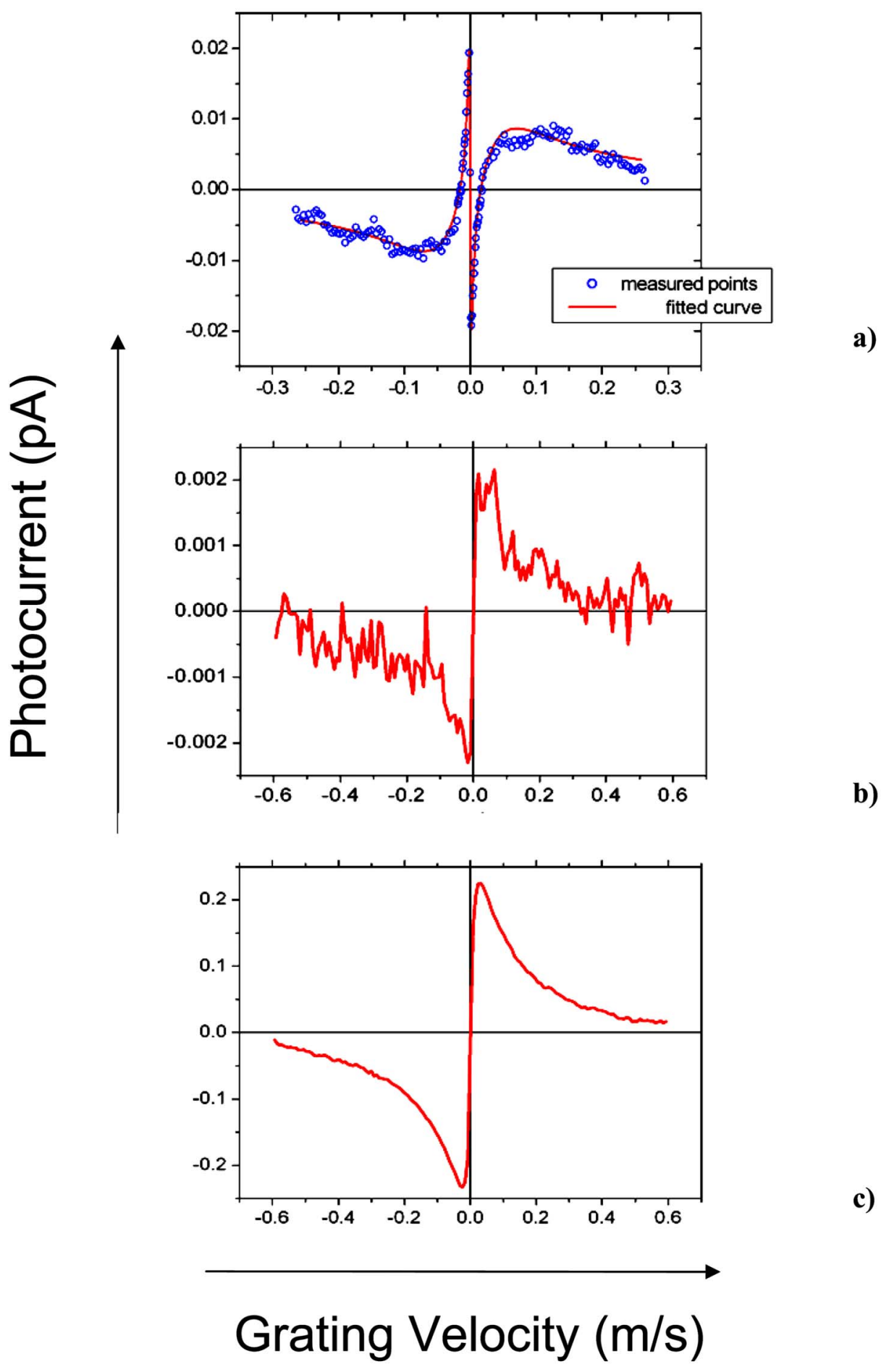

FIG. 5. (Color online) Photocurrent response vs grating velocity for (a) high concentrations of CdTe in MEH-PPV, (b) for pure CdTe nanoparticle films, and (c) for high concentrations of CdTe in polystyrene. In part (a), the pair of narrow peaks is due to the electron transport in the $n$-type $\mathrm{CdTe}$ and the broader peaks due to the hole transport in MEH-PPV. In (b) and (c), the photocurrent response is due to the holes acting as majority carriers in $p$-type CdTe.

c)

transport processes of electrically decoupled electron and hole ensembles, which results from a charge separation at the interface. In each of these ensembles, the unipolar transport conditions prevail with photogenerated excess carrier densities larger than the thermal carrier densities in CdTe or MEH-PPV. The two ensembles give rise to response currents of opposite polarity, which manifest themselves in two current signals of opposite polarity. This situation is clearly different from the conditions in homogeneous materials, where local space charge neutrality produces a coupled, ambipolar transport process. ${ }^{15,16}$
To further investigate the transport in CdTe, we have prepared CdTe nanoparticle films without the surrounding polymer and with a polymer whose HOMO and LUMO energies do not allow any charge transfer from CdTe. In both types of films, the MPG signal is expected to exhibit an ambipolar response only from the $\mathrm{CdTe}$ network. The results for the pure CdTe film are shown in Fig. 5(b). As expected, a single peak set is observed, with the polarity indicating the hole transport to be dominant. The hole conductance is easily explained by the fact that undoped CdTe turns $p$ type with exposure to air, as is the case with the our CdTe nanopowder. 


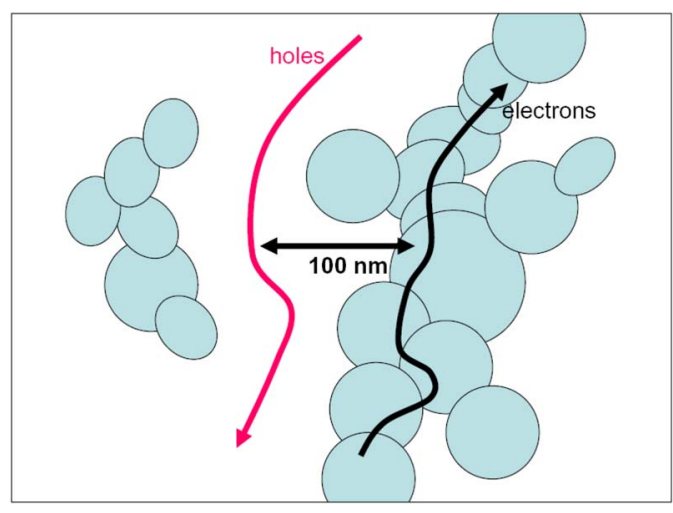

FIG. 6. (Color online) Schematic of the electron and hole transport in the hybrid films with a separation of $100 \mathrm{~nm}$ for the transport paths.

Finding $n$-type conductivity in the CdTe films when the same powder is embedded in MEH-PPV films is just another consequence of the hole transfer process at the CdTe/MEH-PPV interface, which leads to hole depletion and thus turns the nanoparticles $n$-type.

These findings are corroborated by the results in films with a large band gap polymer. Polystyrene with a band gap of $4.8 \mathrm{eV}$ forms a straddling band alignment with CdTe, which allows neither electron nor hole transfer from CdTe to the polymer. In $\mathrm{CdTe} /$ polystyrene films, the photocarrier transport can thus only occur through the nanoparticle network. The results for this case are indeed very similar to those obtained in pure CdTe films and are shown in Fig. 5(c). Again, the transport is hole dominated, and the mobilities and lifetimes are the same as those in the pure CdTe case. Only the current amplitudes in these films are somewhat larger, which is likely due to a better contact between the particles as a result of a wetting effect in the polystyrene matrix. It is thus concluded that the CdTe particles are $p$ type in the pure particle films and in the noninjecting hybrid films, while they turn $n$-type when embedded in MEH-PPV due to a selective hole transfer in the staggered band alignment.

While it has long been recognized that heterojunction materials can enhance the separation probability of photoexcited electron-hole pairs and excitons, the occurrence of unipolar transport in the two transport paths has not yet been observed. This transport mode is likely to occur when the charge separation goes beyond the Debye screening length, which is given by $L_{D}=\left[\varepsilon \varepsilon_{0} k T /\left(n q^{2}\right)\right]^{1 / 2}$, where $\varepsilon_{0}$ is the vacuum permittivity, $\varepsilon$ is the dielectric constant, $k$ is Boltzmann's constant, $n$ is the carrier density, and $q$ is the electronic charge. We can estimate the carrier density in our films from $\Delta n=G \tau$. By using our experimental results, we obtain $L_{D}=100 \mathrm{~nm}$. The average distance between the electron and hole transport paths should thus be larger than $\sim 100 \mathrm{~nm}$ for unipolar conditions to occur. With an average grain size of $\sim 150 \mathrm{~nm}$, these large charge separation distances appear at least possible. Figure 6 shows a schematic of this situation. Of course, the three-dimensional case offers even more possibilities to accommodate the $100 \mathrm{~nm}$ distance in a percolation-type network. Ambipolar transport conditions may be applicable in less coarse geometries wherein the car- rier separation remains within the Debye length. This may be the case in porous semiconductor/electrolyte systems, which are currently discussed in terms of ambipolar concepts, describing the electron transport in the nanoporous material as being screened by the ionic charges in the electrolyte. ${ }^{17,18}$ In these systems, the particle size is of the order of $20-50 \mathrm{~nm}$. The average charge separation distance is then necessarily much smaller than in our films and screening effects remain important. The transport in these systems therefore may remain ambipolar despite the fact that the charge separation is achieved and the recombination is substantially reduced. By including this case into the considerations, one is tempted to suggest that at least three different experimental situations can be realized in "extended junction" films: First, true ambipolar conditions with a coupling through recombination and charge screening as in homogeneous films. Second, a situation of spatial charge separation with a decreased recombination but with a remaining charge coupling, and thirdly, uncoupled unipolar transport conditions as described in this paper.

In many device applications, unipolar transport may offer considerable advantages over ambipolar conditions: Since one carrier type is present at high concentrations while only few counter carriers are present, the recombination kinetics are usually quite different from the ambipolar situation. Typically, the lifetimes for the dominant carrier type are enhanced ${ }^{19}$ and a more complete trap filling is possible. The trap filling in turn can lead to higher carrier mobilities, ${ }^{20}$ faster transport and higher quasi-Fermi-energies, i.e., higher electrochemical potentials. With these desirable properties, device operation can, in principle, be improved beyond what is achievable in homogeneous films.

To conclude, a detailed study of electron and hole transport in CdTe/MEH-PPV hybrid films reveals an effective charge separation at the CdTe/MEH-PPV interface due to the combined effects of band offset and band bending. At low concentrations, the CdTe particles can take the role of an efficient sensitizer to MEH-PPV, producing a photocurrent response at photon energies where MEH-PPV would normally neither have absorption nor photoconductivity. At high concentrations, the nanoparticles can establish a second transport path that is spatially separated and electrically decoupled from the transport path in MEH-PPV. This type of electronic transport system was identified as one out of three systems that can be constructed in extended-junction films. Because the transport is no longer ambipolar, long lifetimes, higher mobilities, and larger electrochemical potentials can be expected. Hybrid films with a staggered band alignment should therefore be quite efficient in separating and transporting photocarriers, as required for detectors, sensors, solar cells, and other devices.

The authors gratefully acknowledge the provision of milled CdTe particles by U. Hommerich at Hampton University, VA. This research was supported by the Oregon Nano and Micro-Technology Institute and by the Office of Basic Energy Research, Department of Energy under Contract No. DE-FG02-07ER46406. 
*rkoe@pdx.edu

${ }^{1}$ Y. Kang, N.-G. Park, and D. Kim, Appl. Phys. Lett. 86, 113101 (2005).

${ }^{2}$ J. Liu, T. Tanaka, K. Sivula, A. P. Alivisatos, and J. M. J. Frechet, J. Am. Chem. Soc. 126, 6550 (2004).

${ }^{3}$ Y. Yin and A. P. Alivisatos, Nature (London) 437, 664 (2005).

${ }^{4}$ W. U. Huynh, J. J. Dittmer, and A. P. Alivisatos, Science 295, 2425 (2002).

${ }^{5}$ J.-Y. Jin, H.-G. Kim, C.-H. Hong, E.-K. Suh, and Y.-S. Lee, Synth. Met. 157, 138 (2007).

${ }^{6}$ U. Hommerich, Physics Department, Hampton University, Hampton, VA.

${ }^{7}$ G. Priebe, B. Pietzak, and R. Könenkamp, Appl. Phys. Lett. 71, 2160 (1997).

${ }^{8}$ U. Haken, M. Hundhausen, and L. Ley, Phys. Rev. B 51, 10579 (1995).

${ }^{9}$ M. Hundhausen and L. Ley, Appl. Phys. Lett. 67, 2518 (1995).

${ }^{10}$ G. Teeter, J. Appl. Phys. 102, 034504 (2007).

${ }^{11}$ N. Dam, M. M. Beerbom, J. C. Braunagel, and R. Schlaf, J. Appl. Phys. 97, 024909 (2005).
${ }^{12}$ Q. Shi, Y. Hou, J. Lu, H. Jin, Y. Li, Y. Li, X. Sun, and J. Liu, Chem. Phys. Lett. 425, 353 (2006).

${ }^{13}$ T. W. Lee, O. Ok Park, J. M. Hong, D. Y. Kim, and Y. C. Kim, Thin Solid Films 393, 347 (2001).

${ }^{14}$ A. R. Inigo, H.-C. Chiu, W. Fann, Y.-S. Huang, U. S. Jeng, C. H. Hsu, K.-Y. Peng, and S.-A. Chen, Synth. Met. 139, 581 (2003).

${ }^{15}$ D. Ritter, E. Zeldov, and K. Weiser, Phys. Rev. B 38, 8296 (1988).

${ }^{16}$ Previous experiments and theory (Refs. 8 and 9) show that for ambipolar conditions, the MPG signal has only one extremum in each branch of the curve and that the polarity of the overall net current is that of the majority carriers. The ambipolar mobility is given by $\mu=\left[\mu_{e} \mu_{p}(p-n)\right] /\left(\mu_{e} n+\mu_{p} p\right)$.

${ }^{17}$ B. O'Regan and M. Graetzel, Nature (London) 353, 737 (1991).

${ }^{18}$ A. Zaban, A. Meier, and B. A. Gregg, J. Phys. Chem. B 101, 7985 (1997).

${ }^{19}$ A. Rose, Concepts in Photoconductivity and Allied Problems (Interscience, New York, 1963).

${ }^{20}$ D. E. Theodorou and H. J. Queisser, Phys. Rev. Lett. 58, 1992 (1987). 